\title{
On a subvariety of the moduli space
}

Francisco Javier Cirre

\begin{abstract}
We give an explicit description of a non-normal irreducible subvariety of the moduli space of Riemann surfaces of genus 3 characterized by a non-cyclic group action. Defining equations of a family of curves representing non-normal points of this subvariety are computed. We also find defining equations of the family of hyperelliptic curves of genus 3 whose full automorphism group is $C_{2} \times C_{4}$. This completes the list of full automorphism groups of hyperelliptic curves.
\end{abstract}

\section{Introduction}

The moduli space $\mathcal{M}_{g}$ of compact Riemann surfaces (or complex algebraic curves) of genus $g \geq 2$ can be viewed as the quotient of the Teichmüller space under the action of the modular group. The action is not fixed point free and therefore $\mathcal{M}_{g}$ has a singularity locus that consists (if $g>2$ ) of the surfaces with non-trivial automorphism group. Certain irreducible subvarieties $\mathcal{M}_{g}(H)$ of this singular locus are studied in [6]. They are characterized by the specification of a finite group $H$ of mapping-classes whose action on a surface is fixed geometrically. In general, these subvarieties fail to be normal. Using arguments on the dimension of the set of non-normal points it is shown in [6] the existence of a non-normal subvariety $\mathcal{M}_{g}(H)$, where $H$ is a cyclic group of order 2. A more explicit example is given in [7], where the authors find defining equations of curves corresponding to non-normal points of a certain subvariety $\mathcal{M}_{g}(H)$, in this case $H$ being a cyclic group of order 8 acting on genus 9. Also in the case of cyclic actions, in [5] it is shown that if $H$ has prime order and the quotient space $X / H$ has genus zero, then topological conjugacy implies conformal conjugacy. Classical results concerning topological conjugacy of cyclic automorphism groups are found in [12], [8] and [4]. Less results are known for non-cyclic actions.

2000 Mathematics Subject Classification: 14H, 30F, 32G.

Keywords: Riemann surface, moduli space, automorphism group. 
In this note we give an explicit description of a subvariety of $\mathcal{M}_{3}$ characterized by a non-cyclic action which has been chosen so that the subvariety is non-normal. We find defining equations of a family of curves which correspond to a one-dimensional submanifold formed by non-normal points of this subvariety.

A basic ingredient to study conformal conjugacy of automorphism groups of a given surface is the knowledge of the full automorphism group of the surface in question. This leads to the problem of extendability of group actions, that is, the problem of deciding whether a finite group acting on a surface is its full automorphism group. This question has been studied in detail for the family of hyperelliptic Riemann surfaces in [2] where, using Fuchsian groups and Teichmüller theory, it is computed the list of groups acting as the full automorphism group of some hyperelliptic surface. However, there is a gap in this list since there it is claimed that $C_{2} \times C_{4}$ never acts as the full automorphism group in genus 3 . We correct this mistake here and describe explicitly the family of genus 3 hyperelliptic Riemann surfaces whose full automorphism group is $C_{2} \times C_{4}$. This completes the list. This family constitutes a one-dimensional submanifold of the non-normal subvariety mentioned above.

\section{Preliminaries}

Let $H_{0}$ be a group of automorphisms of a compact Riemann surface $X_{0}$ of genus $g \geq 2$. A Riemann surface $X$ is said to have $H_{0}$-symmetry if there exists a homeomorphism $\phi: X_{0} \rightarrow X$ such that $\phi H_{0} \phi^{-1}$ is a group of automorphisms of $X$. The set $\mathcal{M}_{g}\left(H_{0}\right)$ of isomorphism classes of Riemann surfaces with $H_{0}$-symmetry is an irreducible subvariety of the moduli space $\mathcal{M}_{g}$ of Riemann surfaces of genus $g$, see [6, Theorem 1]. In general, $\mathcal{M}_{g}\left(H_{0}\right)$ is non-normal. Indeed, it fails to be normal if and only if there exists a Riemann surface $X \in \mathcal{M}_{g}\left(H_{0}\right)$ whose full automorphism group Aut $X$ contains a subgroup $H$ which is topologically but not conformally conjugate to $\phi H_{0} \phi^{-1}$. Riemann surfaces satisfying this property correspond to non-normal points of $\mathcal{M}_{g}\left(H_{0}\right)$.

Every compact Riemann surface can be written as the quotient $U / \Lambda$ of the hyperbolic plane $U$ under the action of a surface Fuchsian group, that is, a Fuchsian group without elliptic elements. A finite group $H$ acts as a group of automorphisms of $U / \Lambda$ if and only if there exist a Fuchsian group $\Gamma$ containing $\Lambda$ as a normal subgroup, and an epimorphism $\theta: \Gamma \rightarrow H$ whose kernel is $\Lambda$. An epimorphism with a surface Fuchsian group as a kernel is called smooth. Every Fuchsian group has a presentation of the following 
form. It has generators $a_{1}, b_{1}, \ldots, a_{\gamma}, b_{\gamma}, x_{1}, \ldots, x_{r}$ and defining relations

$$
x_{1}^{m_{1}}=\cdots=x_{r}^{m_{r}}=1, \quad \prod_{j=1}^{r} x_{j} \prod_{i=1}^{\gamma}\left[a_{i}, b_{i}\right]=1 .
$$

A Fuchsian group $\Gamma$ with this presentation is said to have signature $\sigma(\Gamma)=$ $\left(\gamma ; m_{1}, \ldots, m_{r}\right)$. The integers $m_{i}$ are called proper periods, and $\gamma$ is the genus of the quotient orbifold $U / \Gamma$.

A genus 3 hyperelliptic surface $X$ is represented by $y^{2}=P(x)$ where $P$ is a monic polynomial of degree 7 or 8 with no multiple root. The branch point set $B_{X}$ of $X$ consists of the roots of $P$ if $\operatorname{deg} P=8$ or the roots of $P$ together with $\infty$ if $\operatorname{deg} P=7$. The hyperellipticity of $X$ is characterized by the existence of an involution, called hyperelliptic, which fixes 8 points. It is a central element in the full group Aut $X$ of all automorphisms of $X$, and its formula is $(x, y) \mapsto(x,-y)$. If $Y$ is another such curve then each isomorphism $f: X \rightarrow Y$ induces a unique Möbius transformation $\widehat{f}$ in the Riemann sphere $\widehat{\mathbb{C}}=\mathbb{C} \cup\{\infty\}$ which maps $B_{X}$ onto $B_{Y}$. Conversely, each such a Möbius transformation lifts to two isomorphisms $f_{1}, f_{2}: X \rightarrow Y$ which coincide up to the hyperelliptic involution, see [3]. We will denote by Aut $X_{\widehat{\mathbb{C}}}$ the (finite) group of Möbius transformations induced in $\widehat{\mathbb{C}}$ by the full group Aut $X$ of automorphisms of $X$.

\section{Results}

Let $X_{0}: y^{2}=x^{8}-1$ be the Accola-Maclachlan curve of genus 3 , and let

$$
\text { Aut } X_{0}=\left\langle u, v \mid u^{4}=v^{8}=(u v)^{2}=\left[u^{2}, v\right]=1\right\rangle
$$

be its full automorphism group [1, 10]. It has order 32 and explicit generators

$$
u(x, y)=\left(1 / x, i y / x^{4}\right) \quad \text { and } \quad v(x, y)=\left(x e^{\pi i / 4}, y\right) .
$$

The hyperelliptic involution

$$
u^{2}(x, y)=(x,-y)
$$

and

$$
v^{4}(x, y)=(-x, y)
$$

generate the center of Aut $X_{0}$, which is a non-cyclic group of order 4 .

Theorem 1 The moduli space $\mathcal{M}_{3}\left(\left\langle u^{2}, v^{4}\right\rangle\right)$ of curves with $\left\langle u^{2}, v^{4}\right\rangle$-symmetry is a non-normal irreducible subvariety of $\mathcal{M}_{3}$ of complex dimension 3 , and it consists of the isomorphism classes of the curves given by

$y^{2}=\left(x^{2}-1\right)\left(x^{2}-\alpha_{1}^{2}\right)\left(x^{2}-\alpha_{2}^{2}\right)\left(x^{2}-\alpha_{3}^{2}\right) \quad$ with $\alpha_{i}^{2} \neq \alpha_{j}^{2}$ and $\alpha_{i} \in \mathbb{C}-\{0, \pm 1\}$. 
Proof. Let the (isomorphism class of the) curve $X$ be in $\mathcal{M}_{3}\left(\left\langle u^{2}, v^{4}\right\rangle\right)$ and let $\phi: X_{0} \rightarrow X$ be a homeomorphism such that $\phi H_{0} \phi^{-1}$ is a group of automorphisms of $X$, where $H_{0}=\left\langle u^{2}, v^{4}\right\rangle$. Since the hyperelliptic involution $u^{2}$ fixes 8 points, the same happens to $\phi u^{2} \phi^{-1}$ and so $X$ is hyperelliptic. Analogously, the Möbius transformation induced by $\phi v^{4} \phi^{-1}$ in $\widehat{\mathbb{C}}$ is a rotation (of order 2) which fixes no branch point of $X$. We may suppose that such a rotation is $x \mapsto-x$, and that \pm 1 are branch points of $X$. Therefore, $X$ has the form prescribed in the theorem, and $\mathcal{M}_{3}\left(\left\langle u^{2}, v^{4}\right\rangle\right)$ has complex dimension 3.

We now prove that this space is not normal. Let us consider the group $\left\langle u^{2}, v^{2}\right\rangle \simeq C_{2} \times C_{4}$. Although $\left\langle u^{2}, v^{4}\right\rangle$ is its unique subgroup isomorphic to $C_{2} \times C_{2}$, we shall see that the curves with $\left\langle u^{2}, v^{2}\right\rangle$-symmetry also have another $\left(C_{2} \times C_{2}\right)$-symmetry which, in addition, is topologically but not conformally conjugate to $\left\langle u^{2}, v^{4}\right\rangle$. The point here is that $\left\langle u^{2}, v^{2}\right\rangle$ is not the full automorphism group of a curve with $\left\langle u^{2}, v^{2}\right\rangle$-symmetry, that is, the action of this group on the curve always extends to a larger group. To see this, observe that a curve $X$ with $\left\langle u^{2}, v^{2}\right\rangle$-symmetry is hyperelliptic and its branch point set is preserved by a rotation of order 4 (induced by $v^{2}$ ) which fixes none of them; therefore, it has equation

$$
X_{\lambda}: y^{2}=\left(x^{4}-1\right)\left(x^{4}-\lambda^{4}\right) \quad \text { with } \quad \lambda^{4} \in \mathbb{C}-\{0,1\} .
$$

Clearly, each $X_{\lambda}$ admits the "extra" automorphism

$$
s:(x, y) \mapsto\left(\lambda / x, y \lambda^{2} / x^{4}\right)
$$

which together with $u^{2}$ and $v^{2}$ generate the direct product

$$
\left\langle u^{2}\right\rangle \times\left\langle v^{2}, s\right\rangle \simeq C_{2} \times D_{4} .
$$

This is the full automorphism group of $X_{\lambda}$ for all values of $\lambda$ except for a finite number of them. Indeed, the unique hyperelliptic curves of genus 3 whose full automorphism group contains $C_{2} \times D_{4}$ properly are the Accola-Maclachlan curve, which corresponds to $\lambda^{4}=-1$, and the curves with Aut $X_{\lambda}=C_{2} \times S_{4}$, which are isomorphic to $y^{2}=x^{8}+14 x^{4}+1$ and correspond to $\lambda^{4}=2 \pm \sqrt{3}$. So, except for these values,

$$
\text { Aut } X_{\lambda}=\left\langle u^{2}\right\rangle \times\left\langle v^{2}, s\right\rangle \simeq C_{2} \times D_{4} .
$$

Let us consider the following subgroups of Aut $X_{\lambda}$ :

$$
H_{1}:=\left\langle u^{2}, v^{4}\right\rangle \quad \text { and } \quad H_{2}:=\left\langle u^{2}, s\right\rangle .
$$

Both are isomorphic to $C_{2} \times C_{2}$; however, they are not conjugate within Aut $X_{\lambda}$ since the first one is central. Our purpose is to show that they are topologically conjugate. This will prove the non-normality of the subvariety $\mathcal{M}_{3}\left(\left\langle u^{2}, v^{4}\right\rangle\right)$. 
Let us write $X_{\lambda}=U / \Lambda$ and $\left\langle u^{2}\right\rangle \times\left\langle v^{2}, s\right\rangle=\Gamma / \Lambda$ for some Fuchsian groups $\Lambda$ and $\Gamma$. It is shown in [2] that $\Gamma$ has signature $\sigma(\Gamma)=(0 ; 2,2,2,4)$. Let $\left\{x_{1}, x_{2}, x_{3}, x_{4}\right\}$ be a canonical set of generators for $\Gamma$. After composing with an automorphism on the target, we may suppose that the smooth epimorphism $\theta: \Gamma \rightarrow\left\langle u^{2}\right\rangle \times\left\langle v^{2}, s\right\rangle$ with $\operatorname{ker} \theta=\Lambda$ is given by

$$
\theta\left(x_{1}\right)=u^{2}, \quad \theta\left(x_{2}\right)=u^{2} s v^{2}, \quad \theta\left(x_{3}\right)=s, \quad \theta\left(x_{4}\right)=v^{2} .
$$

Here we are using that $x_{1}, x_{2}$ or $x_{3}$ has to be mapped to $u^{2}$ since otherwise the signature of $\theta^{-1}\left(\left\langle u^{2}\right\rangle\right)$ would not have eight proper periods equal to 2 , as it has to be since $u^{2}$ is the hyperelliptic involution, see [11]. Let us define $\Gamma_{1}:=\theta^{-1}\left(H_{1}\right)$ and $\Gamma_{2}:=\theta^{-1}\left(H_{2}\right)$. Using the Riemann-Hurwitz formula and the fact that $X_{\lambda} / H_{i}$ has genus zero for $i=1,2$, it is easy to see that both groups have the same signature, namely $(0 ; 2,2,2,2,2,2)$. Hence they are isomorphic. Now, any isomorphism $\phi: \Gamma_{1} \rightarrow \Gamma_{2}$ can be realized geometrically, that is, there is a homeomorphism $f$ of the hyperbolic plane such that the conjugation map $\phi_{f}: \gamma_{1} \mapsto f \gamma_{1} f^{-1}$ coincides with $\phi$, see Theorem 3 in [9].

We claim the existence of an isomorphism $\phi: \Gamma_{1} \rightarrow \Gamma_{2}$ such that the corresponding $\phi_{f}$ preserves ker $\theta$. In such a case, $f$ induces a homeomorphism on the surface $U / \operatorname{ker} \theta$ which conjugates $H_{1}$ and $H_{2}$.

To prove the claim let us consider the restriction of $\theta$ to $\Gamma_{1}$. If $\left\{y_{1}, \ldots, y_{6}\right\}$ is a canonical set of generators for $\Gamma_{1}$ then each involution $y_{i}$ has to be conjugate within $\Gamma$ to $x_{1}$ or $x_{4}^{2}$ since $x_{2}$ and $x_{3}$ do not belong to $\Gamma_{1}$, which is normal in $\Gamma$. Those $y_{i}$ conjugate to $x_{1}$ are mapped by $\theta$ to $u^{2}$ since $u^{2}$ is central in $C_{2} \times D_{4}$. For the same reason, those $y_{i}$ conjugate to $x_{4}^{2}$ are mapped by $\theta$ to $v^{4}$. It follows from the relation $y_{1} \cdots y_{6}=1$ that either four or two of the elements $y_{i}$ are mapped to $u^{2}$ (and the other to $v^{4}$ ). The possibility of two elements mapped to $u^{2}$ cannot occur for otherwise the signature of $\theta^{-1}\left(\left\langle u^{2}\right\rangle\right)$ would not have eight proper periods equal to 2 . Therefore, after reordering the generators $y_{i}$ by means of conjugation (see [9]), we may suppose that the restriction of $\theta$ to $\Gamma_{1}$ is given by $\theta\left(y_{i}\right)=u^{2}$ for $1 \leq i \leq 4$ and $\theta\left(y_{i}\right)=v^{4}$ for $i=5,6$. Analogous arguments yield that for some canonical set of generators $\left\{z_{1}, \ldots, z_{6}\right\}$ for $\Gamma_{2}$, the restriction of $\theta$ to $\Gamma_{2}$ is given by $\theta\left(z_{i}\right)=u^{2}$ for $1 \leq i \leq 4$ and $\theta\left(z_{i}\right)=s$ for $i=5,6$. The assignment $y_{i} \mapsto z_{i}$ for $i=1, \ldots, 6$ is an isomorphism $\phi: \Gamma_{1} \rightarrow \Gamma_{2}$, and the corresponding geometrical realization $\phi_{f}$ preserves $\operatorname{ker} \theta$. Indeed, there is an obvious isomorphism

$$
\Phi:\left\langle u^{2}\right\rangle \times\left\langle v^{4}\right\rangle \rightarrow\left\langle u^{2}\right\rangle \times\langle s\rangle
$$

such that $\Phi \circ \theta=\theta \circ \phi_{f}$. Therefore, $H_{1}$ and $H_{2}$ are topologically conjugate, and the theorem is proved. 
Remark 2 We have just seen that the modular subvariety $\mathcal{M}_{3}\left(\left\langle u^{2}, v^{2}, s\right\rangle\right)$ lies in the non-normal locus of $\mathcal{M}_{3}\left(\left\langle u^{2}, v^{4}\right\rangle\right)$. It turns out that $\mathcal{M}_{3}\left(\left\langle u^{2}, v^{2}, s\right\rangle\right)$ is a one-dimensional manifold since it has no non-normal points. Indeed, the unique curves with $\left\langle u^{2}, v^{2}, s\right\rangle$-symmetry whose automorphism groups may admit two non-conjugate subgroups isomorphic to $C_{2} \times D_{4}$ are the Accola-Maclachlan curve $X_{0}$ and the curve whose full automorphism group is $C_{2} \times S_{4}$. However, $\left\langle u^{2}, v^{2}, u v\right\rangle$ is the unique subgroup isomorphic to $C_{2} \times D_{4}$ contained in Aut $X_{0}$, while $C_{2} \times S_{4}$ also contains a unique conjugacy class of subgroups of order 16 by Sylow theorem.

There is another interesting modular subvariety of $\mathcal{M}_{3}\left(\left\langle u^{2}, v^{4}\right\rangle\right)$ consisting also of curves with a $\left(C_{2} \times C_{4}\right)$-symmetry. Namely, $\mathcal{M}_{3}\left(\left\langle u, v^{4}\right\rangle\right)$. Again, the action of $u^{2}$ assures that these curves are hyperelliptic. Unlike the preceding case, this $\left(C_{2} \times C_{4}\right)$-action is non-extendable. In fact, using algebraic equations we show that this is the full automorphism group of all but finitely many of the points in this subvariety. This fills in the gap in [2], where deeper techniques of Fuchsian groups and Teichmüller theory are employed.

Theorem 3 The subvariety $\mathcal{M}_{3}\left(\left\langle u, v^{4}\right\rangle\right)$ consists of the curves of the form

$$
y^{2}=\left(x^{4}-1\right)\left(x^{2}-\omega^{2}\right)\left(x^{2}-1 / \omega^{2}\right) \quad \text { with } \quad \omega\left(\omega^{4}-1\right) \neq 0 .
$$

If $\omega^{12}-193 \omega^{8}-193 \omega^{4}+1 \neq 0$, then $\left\langle u, v^{4}\right\rangle \simeq C_{2} \times C_{4}$ is the full automorphism group of the curve.

Proof. We write $f$ instead of $v^{4}$ to simplify. A curve $X \in \mathcal{M}_{3}(\langle u, f\rangle)$ is hyperelliptic and the induced group of Möbius transformations $\langle u, f\rangle /\left\langle u^{2}\right\rangle \simeq$ $C_{2} \times C_{2}$ can be chosen to be generated by $\widehat{f}: x \mapsto-x$ and $\widehat{u}: x \mapsto 1 / x$. Some of the 8 branch points must be fixed by a rotation in $\langle\widehat{f}, \widehat{u}\rangle$ since otherwise $X$ would be of the form

$$
y^{2}=\left(x^{2}-\alpha^{2}\right)\left(x^{2}-1 / \alpha^{2}\right)\left(x^{2}-\beta^{2}\right)\left(x^{2}-1 / \beta^{2}\right),
$$

which does not admit an automorphism of order 4. Adding up lengths of $\langle\widehat{f}, \widehat{u}\rangle$-orbits gives that there are exactly 4 branch points fixed by elements of $\langle\widehat{f}, \widehat{u}\rangle$. We may suppose that they are the fixed points of $\widehat{u}$ and $\widehat{f} \widehat{u}$. So

$$
X=\left\{y^{2}=\left(x^{4}-1\right)\left(x^{2}-\omega^{2}\right)\left(x^{2}-1 / \omega^{2}\right)\right\} .
$$

Assume now that $\omega^{12}-193 \omega^{8}-193 \omega^{4}+1 \neq 0$. We show that $X$ admits no more automorphisms than those in $\langle u, f\rangle$ or equivalently, that the group Aut $X_{\widehat{\mathbb{C}}}$ of Möbius transformations induced in the sphere, coincides with $\langle\widehat{u}, \widehat{f}\rangle \simeq C_{2} \times C_{2}$. If this is not so then Aut $X_{\widehat{\mathbb{C}}}=A_{4}, A_{5}$, (alternating groups), $S_{4}$ (symmetric group) or $D_{n}$ (dihedral group). 
We first discard the possibility $A_{4}$. An easy computation shows that the rotations $\widehat{m}$ of order 3 such that $\widehat{m} \widehat{f}$ and $\widehat{m} \widehat{u}$ also have order 3 do not preserve the branch point set $B_{X}=\{ \pm 1, \pm i, \pm \omega, \pm 1 / \omega\}$. Therefore Aut $X_{\widehat{\mathbb{C}}}$ cannot contain $A_{4}$ as a subgroup and this also discards the possibilities $S_{4}$ and $A_{5}$. If $\langle\widehat{f}, \widehat{u}\rangle \subset D_{n}$ then the central element of $D_{n}$ would coincide with $\widehat{f}, \widehat{u}$ or $\widehat{u} \widehat{f}$. In these cases, a dihedral group may preserve $B_{X}$ but it is easy to check that this only happens if $\omega^{4}+1=0, \omega^{4}-14 \omega^{2}+1=0$ or $\omega^{4}+14 \omega^{2}+1=0$, respectively, and these are the values excluded in the statement of the theorem.

Remarks 4 (1) The Accola-Maclachlan curve is the only point in $\mathcal{M}_{3}\left(\left\langle u, v^{4}\right\rangle\right)$ with two different conjugacy classes of automorphism subgroups isomorphic to $C_{2} \times C_{4}$. However, they are not topologically conjugate, as can be seen by analyzing the corresponding smooth epimorphisms as in the proof of Theorem 1. Hence, $\mathcal{M}_{3}\left(\left\langle u, v^{4}\right\rangle\right)$ is normal and in fact, a manifold.

(2) The curves described in Theorem 3 are the unique hyperelliptic curves of genus 3 such that Aut $X=C_{2} \times C_{4}$. Indeed, if $X$ is such a curve then either Aut $X_{\widehat{\mathbb{C}}}=C_{2} \times C_{2}$ or $C_{4}$. The first possibility gives curves as in Theorem 3. The second one gives $X=\left\{y^{2}=\left(x^{4}-1\right)\left(x^{4}-\lambda^{4}\right)\right\}$, which we have seen in the proof of Theorem 1 to have an "extra" automorphism.

(3) Unlike $\mathcal{M}_{3}\left(\left\langle u^{2}, v^{2}, s\right\rangle\right)$, the manifold $\mathcal{M}_{3}\left(\left\langle u, v^{4}\right\rangle\right)$ does not lie in the non-normal locus of $\mathcal{M}_{3}\left(\left\langle u^{2}, v^{4}\right\rangle\right)$. However, it lies in the non-normal locus of another subvariety consisting of curves with a cyclic symmetry, namely, $\mathcal{M}_{3}(\langle u\rangle)$ (see [7, Remark 1]).

Acknowledgments: I would like to thank Gabino González-Díez for his patient listening to my questions concerning the non-normality of modular subvarieties, and for answering them all.

\section{References}

[1] Accola, R. D. M.: On the number of automorphisms of a closed Riemann surface. Trans. Amer. Math. Soc. 131 (1968), 398-408.

[2] Bujalance, E., Gamboa, J. M. and Gromadzki, G.: The full automorphism groups of hyperelliptic Riemann surfaces. Manuscripta Math. 79 (1993), 267-282.

[3] Cirre, F. J.: Birational classification of hyperelliptic real algebraic curves. Submitted.

[4] Gilman, J.: On conjugacy classes in the Teichmüller modular group. Michigan Math. J. 23 (1976), 53-63.

[5] GonzÁlez-Díez, G.: On prime Galois coverings of the Riemann sphere. Ann. Mat. Pura Appl. (4) 168 (1995), 1-15. 
[6] González-Díez, G. And Harvey, W. J.: Moduli of Riemann surfaces with symmetry. In Discrete groups and geometry (Birmingham, 1991), 75-93. London Math. Soc. Lecture Note Ser. 173. Cambridge Univ. Press, Cambridge, 1992.

[7] González-Díez, G. And Hidalgo, R.: Conformal versus topological conjugacy of automorphisms on compact Riemann surfaces. Bull. London Math. Soc. 29 (1997), no. 3, 280-284.

[8] Harvey, W. J.: On branch loci in Teichmüller space. Trans. Amer. Math. Soc. 153 (1971), 387-399.

[9] Macbeath, A. M.: The classification of non-euclidean plane crystallographic groups. Canad. J. Math. 19 (1967), 1192-1205.

[10] Maclachlan, C.: A bound for the number of automorphisms of a compact Riemann surface. J. London Math. Soc. 44 (1969), 265-272.

[11] Maclachlan, C.: Smooth coverings of hyperellyptic surfaces. Quart. J. Math. Oxford Ser. (2) 22 (1971), 117-123.

[12] Nielsen, J.: Untersuchungen zur Topologie der geschlossenen zweisetigen Flachen. Acta Math. 50 (1927), 264-275.

Recibido: 28 de octubre de 2002

Francisco Javier Cirre

Departamento de Matemáticas Fundamentales

Facultad de Ciencias

UNED

Madrid 28040, Spain

jcirre@mat. uned.es

Partially supported by BFM2002-04801. 